\title{
EL CULTIVO DEL VIÑEDO COMO RECURSO TURÍSTICO CULTURAL: EL CASO DE LA GERIA (LANZAROTE. ISLAS CANARIAS, ESPAÑA)
}

\author{
Alejandro González Morales, Antonio Ángel Ramón Ojeda y Santiago Hernández \\ Torres \\ Universidad de Las Palmas de Gran Canaria
}

\begin{abstract}
RESUMEN
La agricultura es un sector económico importante pero actualmente en regresión. El paisaje agrícola constituye un patrimonio que puede ser puesto en valor como recurso turístico, de manera que ambas actividades, agricultura y turismo, se verían mutuamente beneficiadas de esa relación. El presente trabajo aborda la evolución y situación actual del sector vitivinícola, su producción y sistemas de cultivo, distribución espacial y expectativas de futuro. Asimismo, se pone en relación el paisaje vitivinícola con la actividad turística y el uso que del primero se está llevando a cabo, y aún puede potenciarse, como atractivo en rutas y actividades de ocio para los visitantes de Lanzarote.
\end{abstract}

Palabras clave: Paisaje agrícola; viticultura; enoturismo; desarrollo local; recurso turístico.

\section{CULTIVATION OF VINEYARDS AS A CULTURALLY TOURIST RESOURCE: THE CASE OF LA GERIA. (LANZAROTE. CANARY ISLANDS, SPAIN)}

\section{ABSTRACT}

Agriculture is an important economic sector but on regression in relation to the past period. The agricultural landscape is a heritage that can be value as a tourist resource, so that both activities, agriculture and tourism, would mutually benefit from that relationship. This paper deals with the evolution and current status of the wine sector, its production and farming systems, spatial distribution and future expectations. Also, placed in relation the wine landscape with tourist activity and use that is being conducted of the first, and may even be enhanced like attractive on routes and recreational activities for visitors to Lanzarote.

Key words: Agricultural landscape; viticulture; tourist resource; wineturist; local development

Fecha de recepción: 18 de mayo de 2015. Fecha de aceptación: 21 de septiembre de 2015. 


\section{INTRODUCCIÓN. CONTEXTO TERRITORIAL Y ESQUEMA METODOLÓGICO DE LA INVESTIGACIÓN}

Lanzarote constituye la más oriental de las Islas Canarias y apenas dista $100 \mathrm{~km}$ del continente africano. Es también una isla baja, que alcanza su mayor altitud en el macizo de Famara al norte de la Isla, teniendo su cumbre en Peñas del Chache, a 670 metros sobre el nivel del mar, circunstancia que reduce la humedad que pudiera aportar el alisio al chocar con el relieve. Tiene una superficie de $850 \mathrm{~km}^{2}$ (906 si incluimos el pequeño archipiélago Chinijo que se localiza al norte de Lanzarote). Es de origen volcánico, comenzando su formación hace unos 15 millones años, cuando se iniciaron las primeras erupciones subaéreas. En 1730 tuvo lugar la mayor erupción histórica de Canarias, la de Timanfaya o Montañas del Fuego, cuyo episodio se prolongó durante seis años, en cinco periodos diferentes. (DE LA HOZ, A., 1994).

El cultivo masivo del viñedo en Lanzarote data del siglo XVIII, tras la conformación de arenados volcánicos surgidos debido a la erupción de Timanfaya (1730-1736). Su mayor superficie se localiza en la zona centro-meridional, donde alcanza una extensión de aproximadamente $200 \mathrm{~km}^{2}$ y conforma un espacio que recibe el nombre de La Geria. Aunque la viña era conocida en la Isla desde el siglo XVI, ésta no conformaba grandes superficies y constituía un cultivo aislado cuya producción era destinada a aguardiente (LOBO CABRERA, 1990). La técnica de cultivo del arenado permitió producir diferentes variedades de viñas (Malvasía, Diego, Listán, Burra...) en un territorio que tiene un clima semidesértico, donde las precipitaciones, irregulares y ocasionalmente torrenciales, rara vez superan los $150 \mathrm{~mm}$ anuales. Además, la evapotranspiración es elevada, entre 700 y $800 \mathrm{~mm}$ al año, y las temperaturas medias rondan $\operatorname{los} 20^{\circ} \mathrm{C}$, siendo el mes más frío enero, cuando las temperaturas no bajan de $10^{\circ} \mathrm{C}$, y el más cálido agosto, cuando se sitúan en torno a los $30^{\circ} \mathrm{C}$. Cabe destacar, por último, que la fuerza y persistencia de los vientos alisios es considerable, superando en ocasiones los $25-30 \mathrm{~km} / \mathrm{hora}$, en especial con la llegada del verano. No obstante, y pese a estas adversas condiciones, los efectos higroscópico, mulching y de cubierta del suelo del lapilli frente a la escorrentía, han propiciado un original paisaje agrario y la posibilidad de desarrollar cultivos realmente viables.

El objetivo de este trabajo es poner de manifiesto que el viñedo insular se puede convertir, y de hecho ya lo es, en un importante recurso turístico que contribuya a dinamizar y diversificar el sector de la explotación del ocio en la Isla, como ha ocurrido en otros lugares (CÁNOVES, G. et al, 2005; GOYET, P. 2010; PARENTEAU, A. 2010). Asimismo, también puede ayudar a relanzar el sector primario, en crisis desde la implantación y desarrollo de la actividad turística con la que entra en competencia, tanto por requerimientos de suelo y agua, como, y sobre todo, por la mano de obra (ALONSO SANTOS J.L., 2003).

Para este trabajo partimos de cuatro premisas o hipótesis iniciales: Lanzarote tiene recursos agrarios suficientes para poder explotar el turismo cultural con absolutas garantías de éxito; el viñedo es un cultivo que puede dinamizar actividades turísticas complementarias al modelo de sol y playa; el mantenimiento del paisaje de La Geria supone incrementar los activos patrimoniales de Lanzarote $\mathrm{y}$, en consonancia, la actividad turística puede contribuir positivamente a su mantenimiento (CÁNOVES VALIENTE, $\mathrm{G}$ et al, 2005).

La metodología del presente trabajo es por un lado hipotético-deductiva, es decir, partimos de unas hipótesis que intentamos demostrar en el desarrollo del trabajo, para finalizar con unas conclusiones. Por otro lado se realizan entrevistas en profundidad a bodegueros y cosecheros, así como a directivos de la denominación de origen, lo cual no aporta un análisis e información cualitativa de gran valor. El estudio tiene también una parte empírica acompañada de aparato cartográfico, gráfico y fotográfico que se ha extraído del material cuantitativo que se manejó para elaborar este estudio.

Las fuentes principales para este trabajo han sido los censos agrarios, los informes del Cabildo Insular, las estadísticas del Cabildo de Lanzarote publicadas en la página web (www.centrodedatos.com), las estadísticas del Gobierno Autónomo de Canarias, los documentos de las bodegas, la consejería de turismo del Cabildo de Lanzarote, las concejalías 
de desarrollo local, turismo y medio ambiente de los ayuntamientos de Teguise, Tinajo y Yaiza y, por último, el Consejo Regulador del vino de Lanzarote.

\section{LOS RASGOS CULTURALES DEL PAISAJE VINÍCOLA. LAS TÉCNICAS Y SISTEMAS DE CULTIVO EN LOS VIÑEDOS DE LANZAROTE}

El viñedo de La Geria se planta en parcelas donde se excavan hoyos (a modo de conos invertidos) en lapilli o bien se realizan zanjas con muros separándolas de tal manera que hacen la función de cortavientos. En los arenados artificiales por lo general se aprovechan los bordes de las fincas para plantar viñedo y en la zona interna se cultivan hortalizas, leguminosas o tubérculos. Las pendientes de los suelos de cultivo no suelen ser muy elevadas, pues cuando ésta se incrementa se realizan trabajos de abancalamiento o terrazas. El viñedo se extiende desde los 200 hasta los 500 metros sobre el nivel del mar. Los suelos son en general de escasa fertilidad (aridisoles y vertisoles). Algunos de estos suelos aparecen fosilizados al haber sido cubiertos por erupciones volcánicas más recientes o por depósitos coluviales. Presentan colores rojizos, textura francolimo-arcillosa, con elevado contenido medio de materia orgánica, lo cual asegura un suministro de nitrógeno adecuado a las exigencias de la vid. Los contenidos de macronutrientes principales $(\mathrm{N}, \mathrm{P}, \mathrm{K}$ ) son óptimos y hay presencia de carbonatos libres que implican un PH básico (Consejo Regulador del Vino, 2014).

Lanzarote cuenta con diversas técnicas y sistemas para cultivar viñedos, ello obedece en gran medida, aunque no de forma exclusiva, a sus singulares características naturales de (geología, clima, suelo,...). Con todo, es la acción del hombre, aprovechando estas ventajas de la naturaleza y sus recursos culturales, la que explica en última instancia el paisaje cultural del vino en Lanzarote.

\subsection{Los arenados naturales}

Los arenados de Lanzarote (figura 1) surgen tras la erupción de Timanfaya en 1730-1736. Este espacio que quedó cubierto por las lapillis del volcán es lo que hoy conocemos como La Geria y tiene una extensión aproximada de $20 \mathrm{Km}^{2}$, situándose en el sector suroccidental de la Isla.

La humedad ambiental contribuye a paliar la falta de precipitaciones en la zona, pues el picón tiene unas vacuolas que facilitan el paso del agua condensada (rocío) y que ésta vaya a parar al suelo. Por ello, cuando retiramos la arena el suelo suele estar mojado. Los beneficios del picón en el cultivo son de índole diversa. En primer lugar cabe citar su carácter higroscópico, pues, el lapilli es capaz de captar directamente del medio el agua y estar húmedo a pesar de no haber llovido en mucho tiempo.

Otro de los efectos beneficiosos es que evita la evaporación, ya que esta capa de rofe aísla el suelo impidiendo que la humedad se escape por evaporación hacia la atmósfera. Esta causa, conjuntamente con la anterior, facilita el cultivo en la zona de productos que por condiciones climáticas no le corresponden, pues son más propios de climas mediterráneos y no subdesérticos como el de Lanzarote.

Un tercer efecto beneficioso es el denominado mulching, que consiste en que la capa de picón hace de colchón aislante para la temperatura, es decir, es capaz durante el día de captar la radiación solar y elevar la temperatura del suelo y, por el contrario durante la noche no pierde la radiación terrestre y así la capa edáfica se mantiene a una temperatura siempre por encima de la ambiental.

$\mathrm{Al}$ aumento de la temperatura contribuye también el hecho de que el color negro del picón absorbe la radiación solar, al tener un albedo muy bajo.

Por último, la capa de lapillis impide que se produzca escorrentía superficial, pues si bien es verdad que en Lanzarote llueve muy poco, cuando lo hace suele ser de forma torrencial, lo que propicia una gran pérdida de suelo si éste no está cubierto de rofe. Por eso en La Geria, al existir una gruesa capa de lapilli, la escorrentía apenas tiene lugar y la pérdida por erosión difusa es 
muy baja. (GONZÁLEZ, A. Y BETANCORT, A., 1989). Toda esta serie de razones son las que explican en última instancia el milagro de la vida y de la agricultura, y en particular del viñedo, en la zona de La Geria.

FIGURA 1

Arenado natural de La Geria (izq) y arenado artificial (dch.).

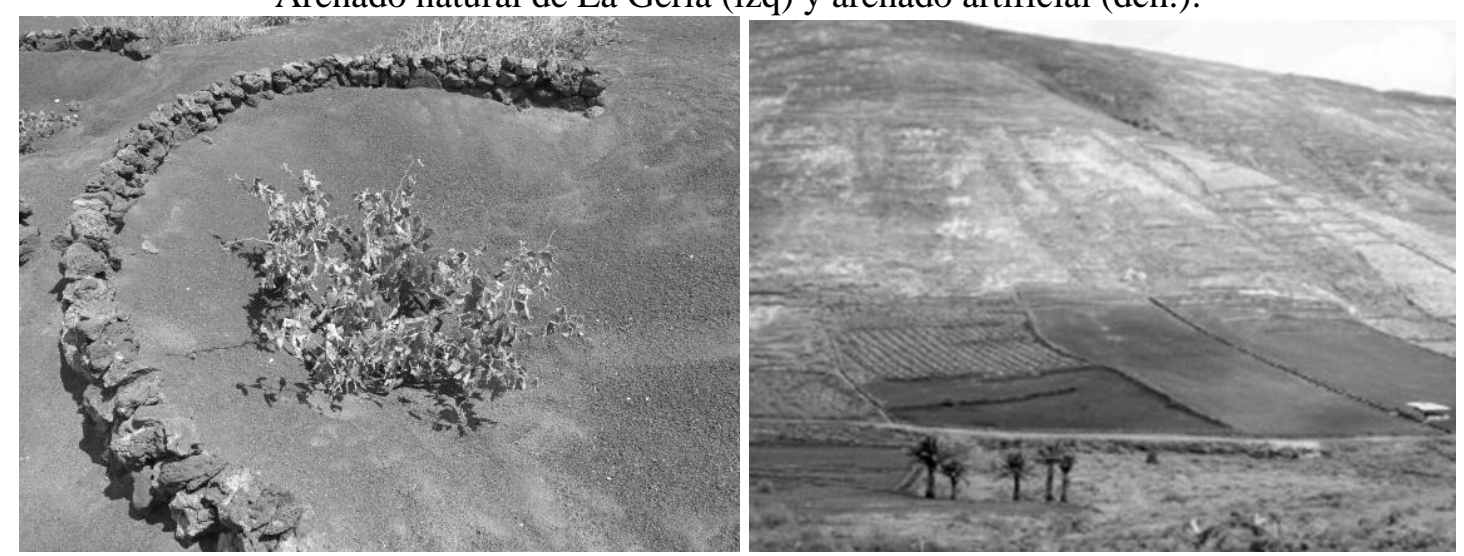

(C) Alejandro González.

\subsection{Los arenados artificiales}

Éstos tienen las mismas propiedades que los anteriores, aunque en esta ocasión la diferencia estriba en que no fue la naturaleza la responsable de su origen, sino que son de factura humana.

$\mathrm{El}$ agricultor aprovecha el momento de su realización para introducir algunas variantes que no tienen los arenados naturales. En efecto, se les dota de una fina capa de estiércol para incrementar la productividad del suelo y también una lámina de polvillo de diez centímetros para impedir que el rofe se mezcle con el estiércol. La capa de picón en los arenados artificiales tiene aproximadamente unos veinte centímetros de espesor.

En 2014, el precio de realización de una hectárea de arenado era de unos $64.520 €$. Siendo los gastos de diversa índole como se aprecia en el esquema de la tabla 1.

El mayor número de arenados en Lanzarote se realizó tras los años cuarenta aprovechando las ayudas del IRIDA (Instituto para la Reforma y el Desarrollo Agrario).

Uno de los casos más llamativos de incremento de arenados se está produciendo con la viña, por el prestigio social que da y por las expectativas que tienen la ayudas europeas para el mantenimiento de estos paisajes dentro de la categoría de Reserva de la Biosfera que en estos momentos tiene la isla de Lanzarote (declarada RB. en 1993).

TABLA 1

Costes de una hectárea de arenado. 2014.

- Tierra Bermeja $\left(1.500 \mathrm{~m}^{3} \times 4 \mathrm{~m}^{2} \times 60 €\right)=22.500 €$

- $\operatorname{Rofe}\left(2.500 \mathrm{~m}^{3} \times 10 \mathrm{~m}^{2} \times 60 €\right)=15.000 €$

- Empolvillado y preparación del terreno $\left(10\right.$ horas tractor x $\left.25 € \ldots 1000 \mathrm{~m}^{2}\right)=2.500 €$

- Estiércol $\left(2 \mathrm{~kg} / \mathrm{m}^{2} \times \quad 0,05 €\right)=1.000 €$

- Paredes $\left(30 \mathrm{~cm} \times 10 \mathrm{~m}\right.$. lineales; $\left.1 \mathrm{~m}^{3}\right)=120 €$

- Trabajo muro $(50 \mathrm{~m}=1$ jornal $=22.500 €)=22.500 €$

- Mano de obra de desripiado, estiércol y otros $=900 €$

- $\quad$ Total $=64.520 €$

Fuente: Cabildo de Lanzarote y Consejo Regulador del vino. 


\subsection{Los cultivos en zanjas o chabocos}

Otra de las variantes de cultivo que se dan en la zona de La Geria, aunque también aparecen en el malpaís de La Corona, son los denominados cultivos en zanjas y chabocos (figura 2). Entre éstos suelen haber numerosas parras de vid.

Esta técnica consiste en romper las coladas de lavas para buscar el suelo vegetal que hay debajo y después proceder a plantar algún frutal tipo higuera, durazno, guayabo, granadero... e incluso viña. Pueden llevar muretes alrededor para evitar que entren animales y mejorar las condiciones para cortar el viento.

FIGURA 2

Cultivo en Chaboco. Masdache, Lanzarote.

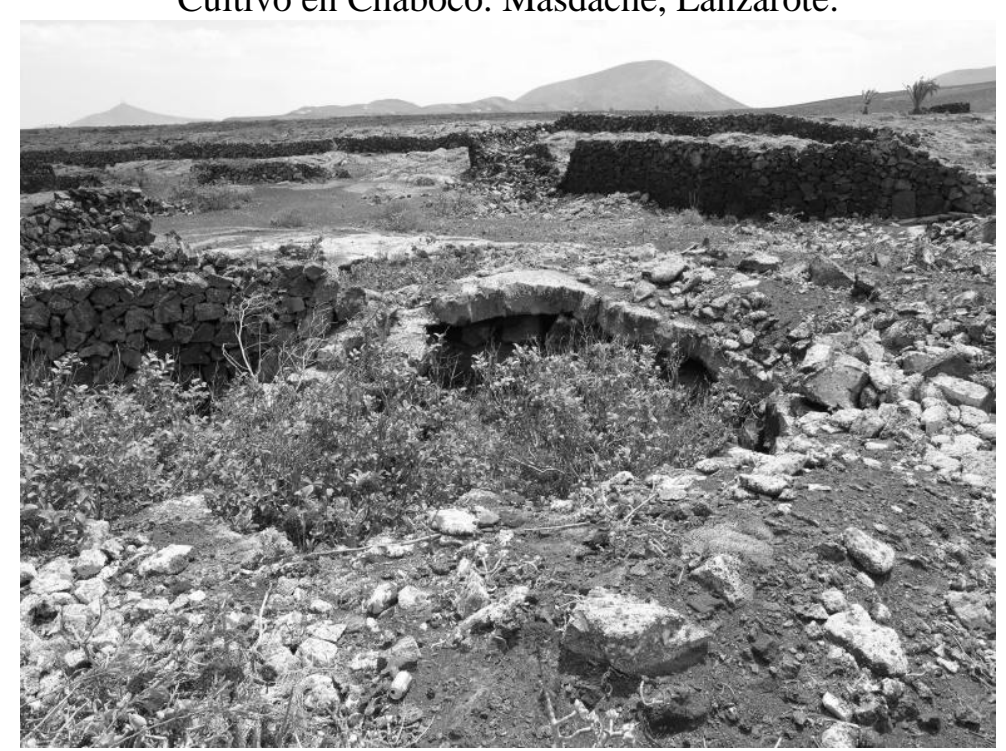

(C)Alejandro González.

\section{EL CULTIVO DE VIÑEDOS EN LANZAROTE Y EN OTROS ÁMBITOS MACARONÉSICOS}

Las técnicas y los sistemas de cultivo del viñedo empleados en Lanzarote son similares a los utilizados en otras islas de la Macaronesia, fuera del ámbito de Canarias, pues la viña se da también en arenados en Pico (Azores), mientras que en Porto Santo (Madeira) el cultivo de este producto se realiza sobre jables (arenas organógenas) (RIBEIRO J., 2000). El jable en la isla de Porto Santo se localiza según el profesor Ribeiro en " El mapa do Porto Santo inclui umas manchas verdes que certifican os terrenos onde estavan cultivadas las vinhas: na Ponta, un pouco afectado das Praia; nas duas margens do Ribeiro Salgado, estendendo-se, na parte oeste,até ao Cabeço da Ponta; duas pequenas manchas atrás do Pico de Ana Ferreira e nas Marinhas; nas duas margens do Ribeiro Cochim, con una cierta incidencia nas Lombas e Madres; toda na zona acima da Vila que se prolongava pelas Areias e que se etrevitava até á Costa Norte; pequeñas parcelas nas Alagoas e muito escassas concentraçoes a Leste da Vila, na Serra da Fora e na Serra de Dentro. Era uma considerável área de vinhas, que foi aumentando até ao final de setecentos (RIBEIRO, J., 2000).

El sistema de cultivo es similar en las tres islas referidas con ligeras variantes que no son dignas de mención. La primera labor que se desarrolla es la plantación, para ello se realiza un hoyo en el lapilli hasta encontrar el suelo vegetal, sobre este último y a una profundidad de 30 $\mathrm{cm}$ aproximadamente se entierra la vara de parra, luego se cubre con la tierra, y sobre ésta se coloca el estiércol, que se renueva cada dos años, o incluso en algunas ocasiones más tiempo. Por último, se cubre de nuevo el suelo con el picón y se realiza un muro de piedra viva alrededor que sirve de cortaviento, o como en el caso de Porto Santo también se pueden emplear 
plantas a modo de setos que evitan el paso de la arena, "As Areias porém, com a acçao do tempo, entravam nas áreas cultivadas, causando muitos danos ás vinhas. Afin de evitarem aquele ermamento causado pela erosao, todos os possuidores de vinhas foram obrigados a fazer plantadas para travarem o avanço das areias. Determinaram ainda que, para evitar a referida invasao das areias, se platassem socas de canas e espinheiros". (RIBEIRO, J., 2000).

Tanto en uno como en otro caso, estamos ante un sistema de cultivo muy original, sobre todo en la zona de arenados naturales (La Geria). La superficie total de viñedo en Lanzarote asciende a unas 3.355 has. (Centro de datos del Cabildo, 2014), que producen caldos de diferentes calidades según las zonas. En este trabajo sólo nos ocuparemos de los viñedos que están dentro de La Geria, pues hay más cultivos de vides, aunque diseminados por el resto de la Isla y de calidades muy dispares, casi todos pertenecientes a bodegas familiares de pequeño tamaño y producción (Información obtenida del trabajo de campo). La viña es el cultivo de mayor extensión en Canarias, y también lo es en Lanzarote. Esta isla cuenta con la segunda mayor extensión de viñedos tras Tenerife, como bien se refleja en el tabla 2.

TABLA 2

Superficie cultivada en Canarias. 2007/2013. Has.

\begin{tabular}{|c|c|c|c|c|c|c|c|c|}
\hline \multirow{2}{*}{$\begin{array}{c}\text { Isla / cultivo / } \\
\text { año }\end{array}$} & \multicolumn{2}{|c|}{ Papas } & \multicolumn{2}{|c|}{ Plátanos } & \multicolumn{2}{|c|}{ Tomates } & \multicolumn{2}{|c|}{ Uvas } \\
\hline & 2007 & 2013 & 2007 & 2013 & 2007 & 2013 & 2007 & 2013 \\
\hline Canarias & 4.262 & 6.699 & 9.563 & 10.589 & 2.260 & 6.840 & 18.934 & 8.094 \\
\hline Lanzarote & 235 & 559 & 3 & 3 & 55 & 74 & 3.078 & 2.292 \\
\hline Fuerteventura & 69 & 12 & - & 24 & 77 & 106 & 50 & 11 \\
\hline Gran Canaria & 1.136 & 1.513 & 1.842 & 2.202 & 1.367 & 3.005 & 401 & 243 \\
\hline Tenerife & 2.191 & 3.817 & 4.242 & 4.849 & 739 & 1.716 & 11.724 & 3.696 \\
\hline La Gomera & 201 & 363 & 165 & 400 & 7 & 35 & 577 & 368 \\
\hline La Palma & 370 & - & 3.250 & - & 14 & - & 2.503 & - \\
\hline El Hierro & 60 & 64 & 60 & 63 & 1 & 8 & 601 & 315 \\
\hline
\end{tabular}

Fuente: Consejería de Agricultura. Gobierno de Canarias.

La productividad en general es baja, siendo el rendimiento por hectárea muy escaso; oscila entre los 1.000 y $1.500 \mathrm{~kg}$. La producción de la Isla está sometida a los antojos climáticos, oscilando entre el millón y los tres millones de kilos ${ }^{2}$ por temporada, con importantes fluctuaciones según el año (Consejo regulador del vino de Lanzarote, 2014). (VV.AA, 1989, 1995, 2007, 2008, 2009).

Las zonas vitivinícolas que se pueden distinguir en Lanzarote son principalmente tres:

1.- La Geria, situada entre el municipio de Yaiza, Tías y Tinajo. El arenado es natural y alcanza hasta cinco metros de profundidad en algunos puntos como resultado de las erupciones volcánicas de 1730-36.

2.- Masdache. Es la zona de mayor extensión y se divide en dos subzonas: la de Tinajo, donde el sistema de cultivo es en zanja y bordes de parcelas; y la de Masdache propiamente dicha, donde predominan los cultivos en hoyos.

3.- Ye - Lajares. Abarca los municipios de Haría y de Teguise. Tiene un suelo de peor calidad y la productividad es menor que en las anteriores localizaciones. Debido a sus condiciones climáticas, se vendimia en el mes de julio, hecho que la convierte en la zona vinícola donde la cosecha es la más temprana de toda Europa (GOYET, P., 2010).

La distribución por municipios del cultivo de la vid aparece recogida en la tabla 3. Las variedades de viña de Lanzarote son: Malvasía, Listán Blanco, Moscatel, Diego, Burra Blanca, Breval, Pedro Ximénez, Listán Negra y Negramoll (tabla 4).

${ }^{2}$ El Consejo regulador del vino de Lanzarote data de diciembre de 1993. Cuenta con 16 bodegas y algo más de 1.700 viticultores. Las bodegas producen 1.500 .000 botellas por año, que se comercializan a partes iguales en el mercado insular y en el exterior (resto de Canarias, Península y algunos países extranjeros, incluso los EE.UU). 
TABLA 3

Número de socios, parcelas y superficie dedicada al viñedo en Lanzarote y controlada por la denominación de origen. 2008.

\begin{tabular}{|l|c|c|c|}
\cline { 2 - 4 } \multicolumn{1}{c|}{} & Socios & $\mathbf{N}^{\mathbf{0}}$ de Parcela & Superf. En has \\
\hline Arrecife & 414 & 1 & 0,5 \\
\hline Haría & 115 & 547 & 226,74 \\
\hline San Bartolomé & 336 & 908 & 288,31 \\
\hline Teguise & 200 & 648 & 121,85 \\
\hline Tías & 231 & 1.050 & 404,75 \\
\hline Tinajo & 356 & 3.093 & 491,26 \\
\hline Yaiza & 48 & 6.821 & 446,38 \\
\hline Otros & 44 & - & - \\
\hline TOTAL & $\mathbf{1 . 7 4 4}$ & $\mathbf{6 . 9 2 9}$ & $\mathbf{1 . 9 7 9 , 7 9}$ \\
\hline
\end{tabular}

Fuente: Consejo Regulador del vino. Lanzarote.

TABLA 4

Distribución por variedades en la isla de Lanzarote. 2014.

\begin{tabular}{|l|r|r|}
\hline \multicolumn{1}{|c|}{ Variedad } & Producción (kg.) & \multicolumn{2}{c|}{ Porcentaje (\%) } \\
\hline Malvasía & 1.046 .994 & 56.07 \\
\hline Listán Negro & 451.338 & 24.17 \\
\hline Diego & 168.959 & 9.05 \\
\hline Moscatel & 127.341 & 6.82 \\
\hline Listán Blanco & 70.514 & 3.78 \\
\hline Burra Blanca & 1.830 & 0.10 \\
\hline Mezcla & 205 & 0.01 \\
\hline
\end{tabular}

Fuente: Consejo Regulador de Vino de Lanzarote. Denominación de Origen. 2014. ${ }^{3}$

La mayor parte del vino lanzaroteño es blanco, bien seco o semiseco, dulce o semidulce; hay poca cantidad de vinos de licor (todos de uva moscatel) y espumosos. De las 3.355 Hectáreas que había en la Isla en 2007, 2.959 son de viñedo plantado como cultivo único en la parcela, mientras que las 403 restantes son de vid asociada a otros cultivos herbáceos (ACOSTA Y FERRER, 2013). La superficie dedicada al cultivo es de 22.000 has, de las cuales unas 8.563,22 son de arenado artificial; 4.770,86 has de empolvillado y 5.222,68 de jable. Ahora bien, para el cultivo de la viña se dedican tan sólo 2.284,10 has en arenado, y unas 1.000 has más de este cultivo asociado a otros herbáceos ${ }^{4}$.

TABLA 5

Producción de uvas de Lanzarote.

\begin{tabular}{|l|r|}
\hline AÑN & \multicolumn{2}{|c|}{ LANZAROTE*(Kilogramos) } \\
\hline 1981 & 2.500 .000 \\
\hline 1991 & 1.555 .700 \\
\hline 2001 & 946.922 \\
\hline 2011 & 716.043 \\
\hline
\end{tabular}

* Sólo están recogidas las producciones que controla el Consejo Regulador. Las producciones artesanales quedan fuera, con lo cual el número total de kilogramos que se produce en la Isla

${ }^{3}$ El Consejo Regulador de vino de Lanzarote comprende las siguientes bodegas: El Grifo S.A., Los Bermejos, Tierra de Volcanes, La Geria S.L., D. Sinforiano Cedrés de Ganzo (Yuco), Rubicón., Castillo Guanapay, Guiguán, El Alto, El Campesino, Bodegas La Grieta, Martinón, Mozaga, Viña Meseta, Vulcano y Stratus. 
está infravalorado.

Fuente: Consejo Regulador del vino. Lanzarote, 1997 -2011.

En la zona norte la densidad de plantación se establece entre 1,5 y 2 m entre cada cepa, mientras que en la de Tinajo-Masdache es de sólo 1,5. Por su parte en La Geria se puede ampliar hasta los cuatro metros entre cepa y cepa. Estas diferencias se deben al diferente sistema de cultivo, pues en La Geria (arenado natural) éste se lleva a cabo en hoyos, y en el resto predomina el de zanja o suelo llano en arenado artificial. Los vinos de las bodegas se comercializan en toda la Isla y en el resto del Archipiélago, aunque de forma excepcional también se han vendido algunas botellas en la Península Ibérica y otros países de Europa, incluso alguna pequeña cantidad se llega a exportar a Estados Unidos. Las restantes bodegas, las que no pertenecen al Consejo Regulador, son de estructura y propiedad familiar y la comercialización de sus vinos sólo se realiza en el mercado local (información de las encuestas en profundidad).

Pese al reconocido prestigio de los caldos lanzaroteños, la producción de uva se ha ido reduciendo de forma muy significativa en los últimos treinta años, pasando de cosechas que en 1981 alcanzaba los dos millones y medios de $\mathrm{kg}$, a tan solo $716.043 \mathrm{~kg}$ en 2011 (tabla 5).

En las figuras 3 y 4 se puede observar la representación gráfica de la distribución del cultivo de la vid en los ámbitos motivo de estudio para el año 2014, según los datos del "Mapa de ocupación del suelo" de Grafcan.

FIGURA 3

Zona vinícola de La Geria (Tinajo-Tías, centro de Lanzarote).

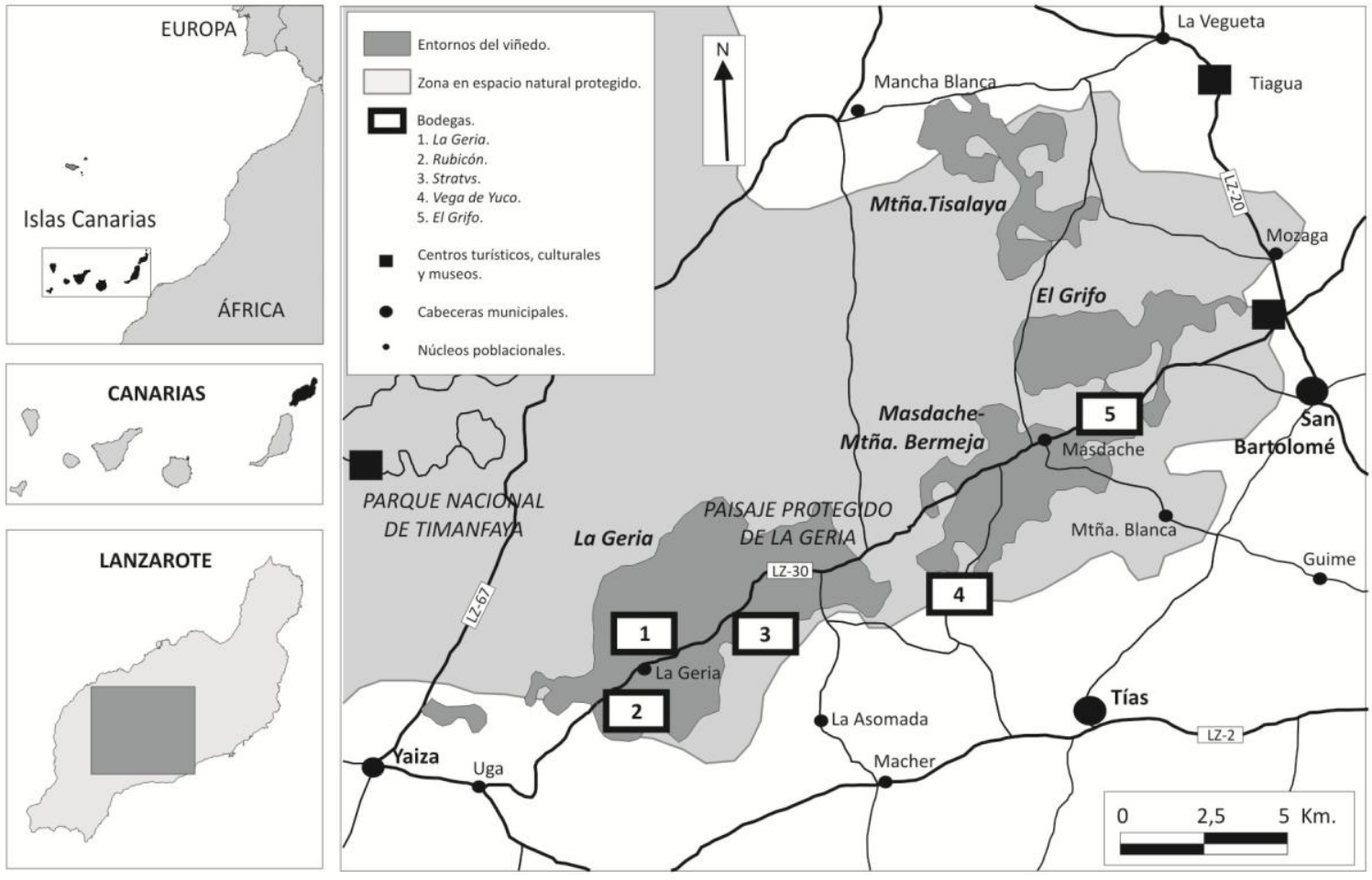

Fuente: MOS. Grafcan. 2014. 
FIGURA 4

Zona vinícola de La Corona (Haría, norte de Lanzarote).
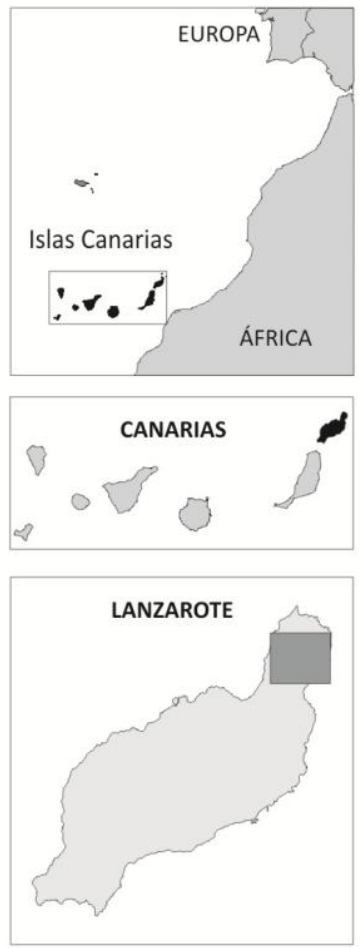

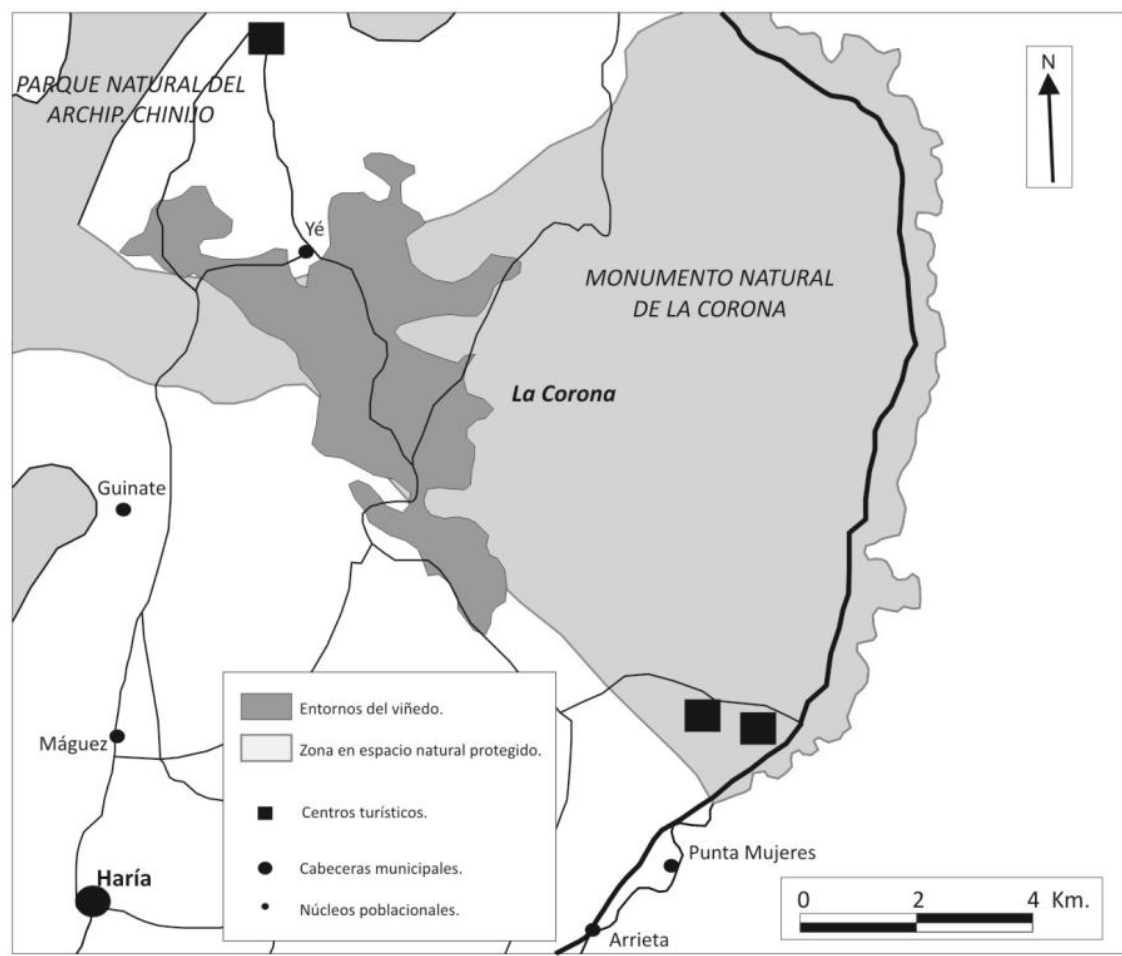

Fuente: MOS. Grafcan. 2014.

\section{LA UTILIZACIÓN TURÍSTICA DE LA GERIA}

La importancia turística de La Geria nadie la discute, precisamente este espacio fue escogido para representar a España como candidatura única en el premio Europeo del paisaje de 2013, un galardón que entrega el Consejo Europeo y que otorgó en dicha edición al Proyecto de revitalización sostenible del Paisaje Protegido de La Geria.

En efecto, La Geria constituye uno de los paisajes más singulares de Lanzarote y también de Canarias y se ha convertido en un espacio de numerosas visitas turísticas, al igual que ocurre con otros lugares de Europa (GOYET, P. 2010, PARENTEAU, A., 2010). Dichas visitas son organizadas tanto por los turoperadores, que incluyen en su programa recorridos por distintas bodegas de la zona (LÓPEZ GUZMÁN-GUZMÁN et al, 2008; MORALES YAGO, F.J., 2012), como también de forma individual y autónoma por parte de muchos turistas que visitan la Isla.

En los últimos años han proliferado las actividades tendentes a utilizar La Geria como un recurso turístico. Por un lado están los itinerarios a pie, a tal efecto encontramos varias rutas, propuestas y preparadas por los ayuntamientos de Tías, Tinajo y Yaiza, y por el Cabildo Insular, que en su Guía Oficial de Senderismo propone una ruta desde el norte de este espacio hasta el sur, atravesándola en su totalidad. Por otro lado están los paseos en bicicleta o a caballo, que también recorren parte de este espacio vinícola. Esta es una manera muy recomendable de apreciar este paisaje, pues no sólo se puede observar el viñedo, sino también otros frutales y cultivos que encierra este espectacular espacio, además del resto del patrimonio cultural del que dispone. Este fenómeno está adquiriendo una gran dimensión en buena parte de España como bien señala Pulido Fernández (PULIDO FERNÁNDEZ, J., et al. 2011).

Las visitas turísticas a las bodegas tiene una doble finalidad, por un lado permitir la compra de vino embotellado con denominación de origen en Lanzarote ${ }^{5}$, o también la de disfrutar de las

\footnotetext{
${ }^{5}$ En el mes de julio se celebra la Semana de los Vinos del Volcán organizada por el Consejo Regulador y donde se realiza una cata de los distintos caldos que produce la Isla de Los Volcanes. Cada año se celebra en un municipio diferente.
} 
exquisiteces gastronómicas que ofrecen los distintos bares y restaurantes asociados a las bodegas. Lanzarote cuenta con una variada oferta gastronómica de pescados (cherne, mero, cabrillas,..), carnes (cabra, oveja, cerdo,..), y cereales y leguminosas (potajes, caldos, pucheros,..), incluso existe una página web (wwwsaborealanzarote.org.) donde se puede consultar la variedad de vinos y las comidas que maridan con ellos. Hay varias bodegas que cuentan con museos o centros de interpretación del vino, como Bodegas El Grifo, Bodegas Rubicón, Bodegas La Geria y Bodegas Stratus (Información obtenida de las encuestas en profundidad). Estas dos últimas son las más recientes, pues no tienen todavía una década de vida, frente a las más antiguas que datan de finales del siglo XVIII y principios del XIX. Una oferta también significativa de La Geria es la pernoctación en casas y hoteles rurales que se hallan en la zona o en sus inmediaciones, siendo la mayoría de ellos establecimientos realizados con muy buen criterio de restauración y puesta en servicio para el turismo rural (Ver oferta alojativa en web del Cabildo de Lanzarote).

Otros aspectos lúdicos relacionados con el turismo y La Geria, son los eventos deportivos, como es la cada vez más famosa carrera del vino (Wine Run Lanzarote), donde los participantes, muchos de ellos turistas extranjeros, degustan los vinos de la zona al mismo tiempo que realizan una actividad deportiva (ACOSTA, T. y FERRER, M, 2013). Otras pruebas deportivas relacionadas con carreras que atraviesan La Geria son el Stratus lavatrail y el internacionalmente conocido Ironman. Asimismo se puede practicar ala delta y parapente desde la montaña próxima de Tinasoria, cerca de las bodegas Rubicón, Chupadero y La Geria.

La Geria acumula también un importante patrimonio arquitectónico y artístico, destacando las construcciones de bodegas del siglo XVIII y XIX como El Grifo y Rubicón respectivamente. También hay numerosas casas rurales de gran valor patrimonial, al igual que dos ermitas: la de La Caridad, frente a la bodega Rubicón, construida en 1706, es decir antes de la erupción de Timanfaya, por mandato del beneficiado D. Diego Laguna Ayala; y la ermita de La Magdalena, en las proximidades de Conil, con un precioso balcón de madera de tea. Interesante construcción artística es el Monumento al Campesino, obra del artista lanzaroteño César Manrique, donde destaca una recreación del hábitat rural insular y una escultura denominada Fecundidad, que simboliza la traída del agua a los campos sedientos de Lanzarote. Hay asimismo interesantes muestras de restos arqueológicos, como por ejemplo el taro de Testeina, estructura habitacional aborigen luego reutilizada por pastores de la Isla, aljibes, pozos, fuentes, chabocos (foto 2), maretas,... (DE LEÓN HERNÁNDEZ, J., 2008).

Por último, hay una serie de museos como el Tanit, en el casco de San Bartolomé, que está dedicado a la etnografía insular y donde se puede apreciar la reconstrucción de una bodega familiar, incluso una prensa de madera de 1780. Otro de los museos relacionados con el mundo rural es El Patio, en el pago de Tiagua, perteneciente al municipio de Teguise, el cual ha sido distinguido con el premio Importante del Turismo, en 2012, por su interesante muestra de la vida tradicional agraria de Lanzarote, evidenciando la importancia del mismo como recurso turístico.

El 15 de agosto se celebra en La Geria, en las inmediaciones de la bodega del mismo nombre, una romería de la vendimia, donde se puede observar los diferentes trabajos que se llevan a cabo en esta actividad agrícola. Por consiguiente, se observa como en La Geria se vienen realizando multitud de actividades relacionadas con el patrimonio cultural, con los deportes o con la gastronomía y que contribuyen a la puesta en valor de este espacio como recurso turístico alternativo.

\section{CONCLUSIONES}

En definitiva, se puede afirmar que los condicionantes naturales no han sido un hándicap para el desarrollo del viñedo en la Isla, más bien todo lo contrario, pues las singulares condiciones de geología y clima han permitido la existencia de unas variedades de uvas y de 
caldos diferentes a los de otros lugares, incluso del propio Archipiélago Canario. En realidad el medio juega un papel importante, pero no deja de ser un mero condicionante, pues son en última instancia el mayor o menor desarrollo de las fuerzas productivas las que posibilitan la ulterior consolidación de las formas de producción. En otras palabras, esta dialéctica que se establece entre el hombre y la naturaleza se zanja con mayor o menor fortuna en función del estadio tecnológico del que dispongan los hombres y de la cantidad de efectivos humanos y capital para poner en funcionamiento los mencionados avances tecnológicos, que por cierto en Lanzarote son escasos, siendo la mayoría de las explotaciones de carácter precapitalista. Por tanto, el paisaje del vino en Lanzarote es un fenómeno cultural donde la acción del hombre ayudado por un medio natural singular y único, ha propiciado unos espacios diferenciados y de gran valor estético y etnográfico.

Respecto a la fuerza de trabajo, podemos afirmar que la Isla ha dejado de ser un espacio agrario para convertirse en un territorio muy terciarizado, donde el turismo es a todas luces la principal actividad y la agricultura ha ido perdiendo protagonismo desde finales de los años sesenta. Ello obedece a que en la Isla de Los Volcanes el sector primario ha dejado de ser el principal en beneficio del secundario (construcción) y, sobre todo del terciario (turismo). Esto ha supuesto que la agricultura en la economía insular pase a tener un papel subsidiario, y la aparición de nuevas fórmulas de trabajo como la agricultura a tiempo parcial sean un hecho constatado. También ha sido necesario introducir las subvenciones para el mantenimiento del subsector agrícola, aunque éstas son muy escasas según los propios agricultores, pero qué duda cabe que han sido fundamentales para el mantenimiento de esta actividad y de este paisaje.

Las técnicas y los sistemas de cultivo producen unos efectos muy beneficiosos, pues no sólo incrementan las condiciones de humedad, sino también de temperaturas, al mismo tiempo que protegen los suelos. La viña en particular es un cultivo que se adapta muy bien a estas condiciones y técnicas y por ello constituye un cultivo destacado en Lanzarote. Las variedades cultivadas varían según las zonas, debido tanto a microclimas locales como a condiciones de suelo y de geología. En efecto, hay diferencias entre La Geria (arenado natural) y la zona de Masdache y de Ye-Lajares, donde el arenado natural se complementa con el artificial.

En lo que respecta a la producción, hay que manifestar que Lanzarote es la que mayor superficie destina al cultivo de la vid en Canarias tras Tenerife y por ello es también la de segunda mayor producción de vinos, aunque en las estadísticas empleadas para esta Isla sólo aparecen las que se encuentran bajo la protección del Consejo Regulador (Denominación de Origen), pues las bodegas familiares han quedado al margen de las mismas. La variedad más cultivada es la malvasía, que se da sobre todo en aquellos municipios que tienen tierras en el espacio protegido de La Geria (arenado natural).

El uso turístico de La Geria es elevado, siendo la fórmula para su aprovechamiento como espacio de ocio muy diversa. El patrimonio cultural de arquitectura y otros elementos artísticos es también considerable lo que coadyuva a incrementar el interés de los turoperadores por este espacio. La oferta vinícola y de gastronomía es diversa, lo cual redunda en la facilidad para su explotación como espacio enológico y gastronómico (ZÁRATE MARTÍN, M. 2010). Por último, señalar que los eventos deportivos, culturales y los alojamientos rurales también están contribuyendo de forma poderosa al uso de La Geria como territorio apto para la actividad turística complementaria al sol y la playa (VELASCO GONZÁLEZ, M., 2009).

\section{BIBLIOGRAFÍA}

ACOSTA, R. Y FERRER, M (2013): Lanzarote y el vino. Paisaje y Cultura. Ediciones Remotas. Arrecife.

ALONSO SANTOS, J.L. (2003) "Redes y procesos de innovación en las comarcas vinícolas de Castilla y León: el ejemplo de la D.O. Bierzo". Boletín de la Asociación de Geógrafos Españoles. № 36. Madrid. pp. 43-60.

CÁNOVES VALIENTE, G., HERRERA JIMÉNEZ, L., VILLARINO PÉREZ, M. 
(2005):"Turismo rural en España: paisajes, nuevos usos y nuevas visiones". Cuadernos de Turismo, $n^{\circ} 15$, pp 63-76.

CÁNOVES, G., HERRERA, L., BLANCO, A. (2005): "Turismo rural en España: un análisis de la evolución en el contexto europeo". Cuadernos de Geografía, no77, pp. 41-58.

CONSEJO REGULADOR DEL VINO DE LANZAROTE (2014): Informe Anual. San Bartolomé. Lanzarote.

DE LA HOZ, A. (1994): Lanzarote. Cabildo Insular de Lanzarote. Colecc. Clásicos de Lanzarote. Vizcaya.

FREITAS CAETANO, S.; RAMÍREZ GARCÍA, S. DEL CANTO FRESNO, C. (2011) Red de relaciones, cohesión social y dinámica territorial. El caso de la Denominación de Origen de Vinos de Uclés (Cuenca).

GONZÁLEZ MORALES, A. ET AL. (2007): Guía oficial de senderos de Lanzarote. Cabildo de Lanzarote.

GONZÁLEZ MORALES, A., (2006): El agua en Lanzarote. Anroart. Las Palmas de Gran Canaria

GONZÁLEZ, A. Y BETANCORT, A., (1989): "Evolución reciente y estado actual de las estructuras agrarias de Lanzarote". II Jornadas de Estudios de Lanzarote y Fuerteventura. Cabildo de Lanzarote. Arrecife.

GOYET, P. (2010) "El turismo vitivinícola: el caso de Francia". Libro de actas del $2^{o}$ Congreso Europeo de Turismo industrial. Toledo 2008. pp. 171-177

GUIRAO PÉREZ, G. (2005): Análisis de las preferencias de los consumidores y de la demanda de vinos en Canarias. Sociedad, Ciencia y Tecnología. Madrid

LOBO CABRERA, M. (1990): “Lanzarote en el siglo XVI. Noticias históricas”. II Jornadas de estudios sobre Lanzarote y Fuerteventura. Tomo I. Cabildo de Lanzarote. Arrecife; págs. 285 300.

LÓPEZ GUZMÁN-GUZMÁN, T.J., SÁNCHEZ CAÑIZARES, S.M. (2008): "La creación de productos turísticos utilizando rutas enológicas". Pasos. Revista de Turismo y Patrimonio Cultural, Vol. 6, $\mathrm{N}^{\mathrm{o}}$ 2, pp 159-171.

MARTÍN MARTÍN, V. (2000): “Aproximación tipológica a los paisajes agrarios actuales de Canarias". Papeles de Geografía, no 32. Universidad de Murcia; p. 97 -115

MATAllanA, A. Y PASCUAL, L. (1992): Denominación de Origen de los vinos de Lanzarote. Excmo. Cabildo Insular de Lanzarote. Arrecife.

MORALES YAGO, F.J. (2012): "Redes y procesos de innovación turística en las comarcas vitivinícolas de la Región de Murcia: Las rutas del vino y sus consecuencias en la transformación del paisaje". Revista de Estudio Andaluces. n 29. pp. 83-104

Ribeiro, J. (2000): Porto Santo. Gobierno de Madeira. Funchal.

VELASCO GONZÁLEZ, M. (2009): "Gestión turística del patrimonio cultural: enfoques para un desarrollo sostenible del turismo cultural". Cuadernos de Turismo, nº 23, pp. 237- 235.

VV. AA (1989): I Jornadas de Estudios de agricultura en la Isla de Lanzarote. La situación insular del cultivo del viñedo. Consejería de Agricultura, Ganadería y Pesca del Cabildo de Lanzarote. Arrecife.

VV. AA (2007): Balance de resultados. Campaña de promoción, comercialización y sensibilización social de los vinos denominación de origen Lanzarote. Cabildo de Lanzarote. Arrecife 
VV. AA. (1995): Análisis del Sector Agrario. Plan Insular de Ordenación del Territorio. Cabildo de Lanzarote.

VV.AA (2008): Análisis monográfico: La importancia de la agricultura y la ganadería en las Canarias del siglo XXI. Consejo Económico y Social. Gobierno de Canarias. Santa Cruz de Tenerife.

VV.AA (2009): Programa de ayudas a la Unión Europea a las producciones agrarias de Canarias. Distribución por Islas y Provincias. Cámara de Comercio. Lanzarote.

VV.AA. (2008): Anuario del Centro de datos del Cabildo Insular de Lanzarote. Cabildo de Lanzarote. Arrecife (www.datosdelanzarote.com).

ZARATE MARTÍN, M.A. (2010):"La “visita de empresa" en España, una modalidad turística en expansión". Libro de actas del $2^{\circ}$ Congreso Europeo de Turismo industrial. Toledo 2008. pp. 52-66. 